\title{
Case Search
}

National Cancer Institute

\section{Source}

National Cancer Institute. Case Search. NCI Thesaurus. Code C127277.

The activity of looking for individuals with a shared characteristic such as a disease. 